H. YAMADA

KODAI MATH. I.

10 (1987), 153-164

\title{
SHARPNESS OF SOME DISTRIBUTIONS ASSOCIATED WITH $A_{m}$-SINGULARITY
}

Dedicated to Professor YoshikazU HiRasawa on his sixtieth birthday

\author{
BY HARUKI YAMADA
}

\section{$\S 1$. Introduction and results.}

In studying sharpness of parametrices for strictry hyperbolic operators, we have encountered some distributions which are defined by the following integrals (see [4]).

$$
G_{q}^{\sigma}(x)=\int_{V} \chi_{q}^{\sigma}(\varphi(x, \theta)) d \theta .
$$

Here $\varphi(x, \theta)$ is a certain polynomial of $(x, \theta)=\left(x_{1}, \cdots, x_{m}, \theta_{1}, \cdots, \theta_{N}\right) \in \boldsymbol{R}^{m} \times \boldsymbol{R}^{N}$ linear in $x$ (a normal form of stable versal singularities; see e.g.[1]), $V$ is a given and fixed neighbourhood of the origin $\theta=0 \in \boldsymbol{R}^{N}$. Further, $\chi_{q}^{\sigma}(\varphi(x, \theta))$ is a distribution defined by $\chi_{q}^{\sigma}(t) \in \mathscr{D}^{\prime}(\boldsymbol{R})$ where

$$
\chi_{q}^{\sigma}(t)=\chi_{q}(t+i 0)+\sigma \chi_{q}(t-i 0), \quad q \in Z / 2, \sigma= \pm 1 .
$$

Here $\chi_{q}(t \pm i 0)=\lim _{\varepsilon \downarrow 0} \chi_{q}(t \pm i \varepsilon) \in \mathscr{D}^{\prime}(\boldsymbol{R})$ are defined by the boundary values on the real axis of the analytic functions

$$
\chi_{q}(z)= \begin{cases}\Gamma(-q) e^{-\pi \imath q} z^{q}, & q \neq 0,1,2, \cdots, \\ z^{q}\left(\log z^{-1}+c_{q}+\pi i\right) / q !, & q=0,1,2, \cdots,\end{cases}
$$

defined on $-\pi<\arg z<\pi$, where $c_{q}=q^{-1}+c_{q-1}$ and $c_{0}=\Gamma^{\prime}(1)$.

The aim of this note is to study sharpness near the origin of the distributions $G_{q}^{\sigma}(x)$ defined above when $\varphi(x, \theta)$ are so called $A_{m}$-singularities, i. e.

$$
\varphi(x, \theta)=\theta^{m+1}+x_{1} \theta^{m-1}+x_{2} \theta^{m-2}+\cdots+x_{m-1} \theta+x_{m},
$$

$(\operatorname{dim} \theta=1)$. More precisely, we shall express a sufficient condition for existence of sharp fronts near the origin by means of a condition for some homology classes (Theorem 2). Further, the condition is restated by means of the behaviour of the roots of equation $\varphi(x, \theta)=0$ under the variation of the parameter $x$ (Theorem 1). As a corollary, we have a sufficient condition for the sharpness

Received May 2, 1986 
of $G_{q}^{\sigma}(x)$ at the origin. Before formulating our theorems, we have to prepare some definitions and propositions.

Definition 1. Let $X \subset \boldsymbol{R}^{n}$ be an open set and $u \in \mathscr{D}^{\prime}(X)$ be a distribution with sing supp $u \subset W$ for some closed set $W \subset X$, where sing supp $u$ is the singular support of $u$. Take and fix a point $x^{0} \in W$ and a component $\omega$ of $X \backslash W$ with $x^{0} \in \partial \omega$. Then $u$ is said to be sharpe at $x^{0}$ from $\omega$ if there is a neighbourhood $U$ of $x^{0}$ and a function $\tilde{u} \in C^{\infty}(U)$ such that $u=\tilde{u}$ on $\omega \cap U$. If we replace sing supp $u$ by $\operatorname{sing} \operatorname{supp}_{A} u$, that is, analytic singular support of $u$, and $C^{\infty}(U)$ by $C^{\omega}(U)$, we can define $C^{\omega}$-sharpness in the same manner.

In what follows we shall concern the $C^{\omega}$-sharpness of $G_{q}^{\sigma}(x)$ though the original problems were concerned the $C^{\infty}$-sharpness. Of course the $C^{\omega}$-sharpness implies the $C^{\infty}$-sharpness.

At first we remark that it is easy to see that

$$
\left(\frac{\partial}{\partial x_{m}}\right)^{k} G_{q}^{\sigma}(x)=G_{q-k}^{\sigma}(x), k=1,2,3, \cdots
$$

Since the sharpness is invariant under the partial differentiations, we can assume that, without loss of generality, that $q$ are negative (half) integers, i.e. $q \in$ $-N / 2$. Further, in $\S 2$, we show

PRoposition 1. Let $V \subset \boldsymbol{R}$ be a fixed nerghbourhood of the origin $0 \in \boldsymbol{R}$. Then there is a neighbourhood $X \subset \boldsymbol{R}^{m}$ of the origin $0 \in \boldsymbol{R}^{m}$ such that

$$
\int_{V} \chi_{q}^{\sigma}(\varphi(x, \theta)) d \theta \equiv \int_{R} \chi_{q}^{\sigma}(\varphi(x, \theta)) d \theta \quad \bmod C^{\omega}(X) .
$$

(The convergence of the right hand integral is guaranteed by the fact that $q \in-N / 2$ and $m \geqq 2$ ). Thus, for studying the sharpness of (1) near the origin, it is enough that we assume $V=\boldsymbol{R}$ and $X=\boldsymbol{R}^{m}$. In what follows we shall therefore assume them and shall consider, instead of (1), the integral

$$
G_{q}^{\sigma}(x)=\int_{\boldsymbol{R}} \chi_{q}^{\sigma}(\varphi(x, \theta)) d \theta \in \mathscr{D}^{\prime}\left(\boldsymbol{R}^{m}\right) .
$$

Then, we have, in $\S 2$, where

Proposition 2. sing $\operatorname{supp}_{A} G_{q}^{o} \subset W$,

$$
W=\left\{x \in \boldsymbol{R}^{m} ; \varphi(x, \theta)=\frac{\partial \varphi}{\partial \theta}(x, \theta)=0 \text { for some } \theta \in \boldsymbol{R}\right\} .
$$

Take a point $x^{0} \in W$ and a component $\omega$ of $X \backslash W=\boldsymbol{R}^{m} \backslash W$ with $x^{0} \in \partial \omega$. We want to make a criterion for sharpness of $G_{q}^{\sigma}(x)$ at $x^{0}$ from $\omega$. In doing so, we have to represent $G_{q}^{\sigma}(x)$ as complex integrations. Let 
$\hat{V}_{x}:=C \backslash\{\theta ; \varphi(x, \theta)=0\}$,

$\tilde{V}_{x}:=\{$ the Riemann surface of the function $\eta=\sqrt{\varphi(x, \theta)}\} \backslash\{\theta ; \varphi(x, \theta)=0\}$.

Note that $\tilde{V}_{x}$ is a double covering of $\hat{V}_{x}$. In $\S 2$, we shall prove

Proposition 3. Take and fix a point $x \in \boldsymbol{R}^{m} \backslash W$ and define chains $\gamma_{\sigma}(x)$ (resp. $\left.\tilde{\gamma}_{\sigma}(x)\right)$ on $\hat{V}_{x}$ (resp. $\tilde{V}_{x}$ ) as Definition 2 in $\S 2$. Then there is a neighbourhood $U$ of $x$ such that

$$
\begin{aligned}
G_{q}^{\sigma}(y)= & \int_{\gamma_{\sigma}(x)} \chi_{q}(\varphi(y, \theta)) d \theta \text { if } q=-1,-2, \cdots \\
& \left(\operatorname{resp} . \int_{\tilde{r}_{\sigma}(x)} \chi_{q}(\varphi(y, \theta)) d \theta \text { if } q=-\frac{1}{2},-\frac{3}{2}, \cdots\right) .
\end{aligned}
$$

In $\S 3$, as Theorem 2 , we shall express our criterion for sharpness by means of some homology classes. Since the definition of our chains $\gamma_{\sigma}(x), \tilde{\gamma}_{\sigma}(x)$ and the formulation of the criterion by the homology classes are somewhat long, we shall postpone them until $\S 3$. However from Theorem 2, we have a definite criterion which will be illustrared in the following manner.

When $x \notin W$, the equation $\varphi(x, \theta)=0$ of $\theta$ has no real multiple roots. If $x$ approach a point $x^{0} \in W$ along a path in $\boldsymbol{R}^{m} \backslash W$, some roots will be confluent and form some multiple real roots. This phenomena are expressed by the combinations of the following five matters.

[ I ] One of $\Phi_{+}[x]$ collapses (i. e. two distinct real roots confluent) and a real multiple root appear.

[II] One of $\Phi_{-}[x]$ collapses and a real multiple root appear.

[III] Two conjugate imaginary roots meet on $\Phi_{+}[x]$ and a real multiple root appear.

[IV] Two conjugate imaginary roots meet on $\Phi_{-}[x]$ and a real multiple root appear.

[V] Two conjugate imaginary roots meet to some real root.

Here we have used the notation

$$
\Phi_{ \pm}[x]:=\{\theta \in R ; \varphi(x, \theta) \gtrless 0\},
$$

which are, if $x \notin W$, union of finite number of discrete open intervals in $\boldsymbol{R}$.

Now we have the following theorem.

THEOREM 1. Let $x^{0} \in W$ and $\boldsymbol{\omega}$ be a component of $\boldsymbol{R}^{m} \backslash W$ with $x^{0} \in \partial \omega$. Then in the following cases, $G_{q}^{\sigma}(x)$ are sharp at $x^{0}$ from $\omega$. 


\begin{tabular}{|l|l|l|}
\hline \multicolumn{1}{|c|}{$q$} & $\sigma$ & change of the roots when $x \rightarrow x^{0}$ in $\omega$. \\
\hline integer & + & only the combinations of [I ] and [II] \\
\hline integer & - & only the combinations of [III] and [IV] \\
\hline half-integer & + & only the combinations of [I] and [IV] \\
\hline half-integer & - & only the combinations of [II] and [III] \\
\hline
\end{tabular}

COROLLARY. If one of the following conditions are satısfied, $G_{q}^{\sigma}(x)$ is sharp at the origin from a component $\omega$ of $\boldsymbol{R}^{m} \backslash W$.

(a) $q \in Z, \sigma=1$ and all the roots of $\varphi(x, \theta)=0$ are real for some (hence for all) $x \in \omega$.

(b) $q \in Z, \sigma=-1$ and all the roots of $\varphi(x, \theta)=0$ are non-real for some (hence for all) $x \in \omega$.

Remark. In connection with parametrices of hyperbolic operators, the condition (b) corresponds to what is called "a trivial lacuna" and the condition (a) corresponds to a non-trivial one.

In $\S 2$, we will representate $G_{q}^{\sigma}(x)$ by complex integrations and prove Propositions 1,2 and 3 . In $\S 3$, we will formulate criterions for sharpness and prove Theorems 1 and 2 .

This work was supported by Grant-in-Aid for Scientific Research from the Ministry of Education, Science and Culture (No. 60540080).

\section{§2. Representation of $G_{q}^{\sigma}(x)$ by a complex integration.}

In this section we shall represent $G_{q}^{\sigma}(x)$, when $x \in \boldsymbol{R}^{m} \backslash W$, as a complex integration on a chain in the $\theta$-space $\boldsymbol{C}$ (Proposition 3 ). In doing so, we have to study the behaviour of the roots of $\varphi(x, \theta)+i \varepsilon=0$ when $\varepsilon$ are small (Lemma $1)$, and we have to define chains $\gamma_{\sigma}(x)$ and $\tilde{\gamma}_{\sigma}(x)$ which have a kind of stability under small perturbations of $x$ (Definition 2). Before doing so, we show that the nature of the integral (1) as a germ at $x=0$ is independent to the choice of the neighbourhood $V$ of $\theta=0$ (Proposition 1), and decide the singularities of the integral (3) (Proposition 2). Though both of them seems to be well known and the proofs are easy, we shall add them for the completeness.

Proof of Proposition 1. The equation $\varphi(0, \theta)=0$ has the $(m+1)$-th multiple roots $\theta=0$. Hence if we fix a complex neighbourhood $\hat{V} \subset C$ of $V$ with $\hat{V} \cap \boldsymbol{R}=V$, we can take a neighbourhood $X \subset \boldsymbol{R}^{m}$ of $x=0$ such that

$$
x \in X \Longrightarrow\{\theta \in C ; \varphi(x, \theta)=0\} \subset \hat{V} \text {. }
$$

Thus, if we take smaller $X$ if necessary, there is a number $\varepsilon_{0}>0$ such that 


$$
x \in X,|\varepsilon|<\varepsilon_{0} \Longrightarrow\{\theta \in C ; \varphi(x, \theta)+\imath \varepsilon=0\} \subset \hat{V} .
$$

Then we have

$$
\begin{array}{r}
\int_{R} \chi_{q}^{\sigma}(\varphi(x, \theta)) d \theta-\int_{V} \chi_{q}^{\sigma}(\varphi(x, \theta)) d \theta=\int_{R \backslash V} \chi_{q}^{\sigma}(\varphi(x, \theta)) d \theta \\
=\lim _{\varepsilon \downarrow 0} \int_{R \backslash V}\left\{\chi_{q}(\varphi(x, \theta)+i \varepsilon)+\sigma \chi_{q}^{\sigma}(\varphi(x, \theta)-i \varepsilon)\right\} d \theta .
\end{array}
$$

The right hand side belongs to $C^{\omega}(X)$ and this proves the proposition.

Remark. The right hand integral of (3) does not necessarily convergent for arbitrary $q \in \boldsymbol{Z} / 2$. But we can extend $G_{q}^{\sigma}(x)$ to $q>0$ by analytic continuation with respect to $q$. These distributions coincide with $(1)$ modulo $C^{\omega}(X)$.

Proof of Proposition 2. Suppose $x^{0} \notin W$. If we take a sufficiently small neighbourhood of $x^{0}$ such that $U \subset \boldsymbol{R}^{m} \backslash W$, we have

$$
\varphi(x, \theta) \neq 0 \text { or } \frac{\partial \varphi}{\partial \theta}(x, \theta) \neq 0 \text { for any } x \in U \text { and any } \theta \in \boldsymbol{R} .
$$

Since $\varphi(x, \theta)=0$ has only finite number of real roots, we can suppose that there are finite number of intervals $V_{k}$ in $\boldsymbol{R}$ such that

(i) $\boldsymbol{R}=\bigcup_{k} V_{k}$,

(ii) for each $k$, there is $\theta_{k}^{0} \in V$ such that

$$
\begin{aligned}
& \varphi\left(x^{0}, \theta_{k}^{0}\right)=0, \frac{\partial \varphi}{\partial \theta}\left(x^{0}, \theta_{k}^{0}\right) \neq 0, \\
& \varphi\left(x^{0}, \theta\right) \neq 0 \quad \text { for all } \theta \in V_{k} \backslash\left\{\theta_{k}^{0}\right\} .
\end{aligned}
$$

Then, in a neighbourhood of $\left(x^{0}, \theta_{k}^{0}\right)$, we can solve $\eta=\varphi(x, \theta)$ with respect to $\theta$; that is, there is a neighbourhood $W_{k} \subset V_{k}$ of $\theta_{k}^{0}$, neighbourhood $T_{k}$ of $\eta=0$, neighbourhood $U_{k} \subset U$ of $x^{0}$ and a function $\phi(x, \eta) \in C^{\omega}\left(U_{k} \times T_{k}\right)$ with valces in $W_{k}$, such that $\varphi(x, \phi(x, \eta)) \equiv \eta$ on $U_{k} \times T_{k}$. Then if we note that $\varphi(x, \theta) \neq 0$ on $U_{k} \times\left(V_{k} \backslash W_{k}\right)$, we have

$$
\begin{aligned}
\int_{V_{k}} \chi_{q}^{\sigma}(\varphi(x, \theta)) d \theta & \equiv \int_{W_{k}} \chi_{q}^{\sigma}(\varphi(x, \theta)) d \theta \quad \bmod C^{\omega}\left(U_{k}\right) \\
& =\int_{T_{k}} \chi_{q}^{\sigma}(\eta) \frac{\partial \varphi}{\partial \theta}(x, \phi(x, \eta)) d \eta
\end{aligned}
$$

Since the right hand side belongs to $C^{\omega}\left(U_{k}\right)$, we have

$$
G_{q}^{\sigma}(x)=\int_{R} \chi_{q}^{\sigma}(\varphi(x, \theta)) d \theta \equiv \sum_{k} \int_{V_{k}} \chi_{q}^{\sigma}(\varphi(x, \theta)) d \theta \in C^{\omega}\left(\bigcap_{k} U_{k}\right) .
$$

Thus $G_{q}^{\sigma}(x)$ is of $C^{\omega}$-class in a neighbourhood of $x^{0}$ and this proves the proposition. 
Now we shall examine the behaviour of the roots of $\varphi(x, \theta)+i \varepsilon=0$ when $\varepsilon$ are small. By the definition of $W$, the equation (2) should not have real multiple roots as long as $x \in \boldsymbol{R}^{m} \backslash W$. Therefore we can arrange the roots $\boldsymbol{\theta}_{j}(x)(j=1,2, \cdots, m+1)$ as follows;

$$
\begin{aligned}
& \theta_{1}(x), \cdots, \theta_{2 s}(x) \text { : conjugate imaginary roots } ; \\
& \quad \theta_{2 j}(x)=\overline{\theta_{2 \jmath-1}(x)}, \operatorname{Im} \theta_{2 \jmath-1}(x)>0, j=1, \cdots, s, \\
& \theta_{2 s+1}(x)<\theta_{2 s+2}(x)<\cdots<\theta_{m+1}(x) \text { : real (distinct) roots. }
\end{aligned}
$$

To represent $G_{q}^{\sigma}(x)$, when $x \in \boldsymbol{R}^{m} \backslash W$, by a complex integration, we have to study the behaviour of the roots $\theta_{j}^{\varepsilon}(x)(j=2 s+1, \cdots, m+1)$ of the equation

$$
\varphi(x, \theta)+i \varepsilon=0
$$

for small $\varepsilon$. Clearly, (4) has no real roots provided that $\varepsilon \neq 0$.

Lemma. Let $x \in \boldsymbol{R}^{m} \backslash W$ and let $\theta_{j}^{\xi}(x)(j=2 s+1, \cdots, m+1)$ be the simple roots of (4) which tend to $\theta_{j}(x)$ as $\varepsilon \rightarrow 0$. Then we have

$$
\begin{aligned}
& \operatorname{Im} \theta_{m+1}^{\varepsilon}(x) \gtrless 0 \quad \text { for small } \varepsilon \gtrless 0, \\
& \operatorname{Im} \theta_{j}^{\varepsilon}(x) \cdot \operatorname{Im} \theta_{j+1}^{\varepsilon}(x)<0 \text { for small } \varepsilon \neq 0, j=2 s+1, \cdots, m .
\end{aligned}
$$

Proof. If we take the derivative of $\varphi\left(x, \theta_{j}^{\varepsilon}(x)\right)+i \varepsilon=0$ with respect to $\varepsilon$ at $\varepsilon=0$, we have

$$
\left.\frac{\partial \varphi}{\partial \theta}\left(x, \theta_{j}(x)\right) \cdot \frac{\partial \theta_{j}^{\varepsilon}(x)}{\partial \varepsilon}\right|_{\varepsilon=0}+\imath=0
$$

Since $\frac{\partial \varphi}{\partial \theta}\left(x, \theta_{j}(x)\right)$ is real, we have

$$
\left.\operatorname{Im} \frac{\partial \theta_{j}^{\varepsilon}(x)}{\partial \varepsilon}\right|_{\varepsilon=0} \gtrless 0 \quad \text { if } \quad \frac{\partial \varphi}{\partial \theta}\left(x, \theta_{j}(x)\right) \lessgtr 0 .
$$

On the other hand, it is clear that

$$
\begin{aligned}
& \frac{\partial \varphi}{\partial \theta}\left(x, \theta_{m+1}(x)\right)>0, \\
& \frac{\partial \varphi}{\partial \theta}\left(x, \theta_{j}(x)\right) \cdot \frac{\partial \varphi}{\partial \theta}\left(x, \theta_{j+1}(x)\right)<0, \quad j=2 s+1, \cdots, m .
\end{aligned}
$$

This proves the lemma.

From the above lemma and the definition of $\chi_{q}^{\sigma}(t)$, we are led to the following definition.

Definition 2. Let us fix a point $x \in \boldsymbol{R}^{m} \backslash W$ and let $\hat{V}_{x}$ and $\tilde{V}_{x}$ be as in $\S 1$. Then 
(i) In $\hat{V}_{x}$, we define the chain $\gamma_{\sigma}(x)$ by

$$
\gamma_{\sigma}(x):= \begin{cases}\sum_{j=1}^{2 s}(-1)^{\jmath+1} \gamma_{j}(x) & \text { if } \quad \sigma=1, \\ \sum_{j=2 s+1}^{m+1}(-1)^{m-j+1} \gamma_{j}(x) & \text { if } \quad \sigma=-1,\end{cases}
$$

where $\gamma_{j}(x)$ is a cycle which is taken to be a simple contour enclosing the point $\theta_{j}(x)$ in the positive direction.

(ii) In $\tilde{V}_{x}$, we define the chain $\tilde{\gamma}_{\sigma}(x)$ by

$$
\tilde{\gamma}_{\sigma}(x):= \begin{cases}\sum_{j=0}^{[(m-2 s) / 2]}(-1)^{j} \tilde{\zeta}_{m-2 j+1}(x) & \text { if } \quad \sigma=1, \\ \sum_{j=0}^{[(m-2 s-1) / 2]}(-1)^{j} \tilde{\zeta}_{m-2 j}(x) & \text { if } \quad \sigma=-1,\end{cases}
$$

where $\zeta_{j}(x)$ is a lift of the chain $\zeta_{j}(x)$ in $\hat{V}_{x}$ to $\tilde{V}_{x}$. Here $\zeta_{j}(x)$ is a cycle which is taken to be a simple contour enclosing the points $\theta_{j}(x)$ and $\theta_{j+1}(x)(j=2 s+$ $1, \cdots, m)$ in the positive direction, and $\zeta_{m+1}(x)$ (resp. $\zeta_{2 s}(x)$ ) is one taken to be a contour which surrounds the part $\left\{\theta \in \boldsymbol{R} ; \theta \geqq \theta_{m+1}(x)\right\}$ (resp. $\left\{\theta \in \boldsymbol{R} ; \theta \leqq \theta_{s+1}\right.$ $(x)\})$ of the real axis in the positive direction and approaches this axis asymptotically on either side. The branch of $\xi_{j}(x)$ are taken so that $\varphi(x, \theta)^{q}$ becomes positive real number when $\tilde{\zeta}_{j}(x)$ crosses the real axis in the positive direction.

Remark. Since $\tilde{V}_{x}$ is a part of the Riemann surface of the hyperelliptic curve $\eta^{2}=\varphi(x, \theta)$, the chains $\tilde{\zeta}_{j}(x)(j=2 s+1, \cdots, m)$ determine closed curves (i. e. cycles) in $\tilde{V}_{x}$. Though two lifts of $\zeta_{j}(x)$ are homotopic in the curve $\eta^{2}=$ $\varphi(x, \theta)$, we have to distinguish them because we deal functions which have singularities at branch points $\theta_{j}(x)$.

Under the above definition we can state and prove Proposition 3.

Proof of Proposition 3. Since the equation (4) has no real roots provided that $\varepsilon \neq 0$, the integral

$$
I_{\varepsilon}(x):=\int_{R} \chi_{q}(\varphi(x, \theta)+i \varepsilon) d \theta+\sigma \int_{R} \chi_{q}(\varphi(x, \theta)-i \varepsilon) d \theta
$$

is a convergent one. Here we use the lemma to deform the path of integration as in [Fig. 1]. Then we have for small $\varepsilon>0$,

$$
I_{\varepsilon}(x)= \begin{cases}\int_{r_{\sigma}(x)} \chi_{q}(\varphi(x, \theta)+i \varepsilon) d \theta & \text { if } \quad q=-1,-2, \cdots \\ \int_{\tilde{r}_{\sigma}(x)} \chi_{q}(\varphi(x, \theta)+i \varepsilon) d \theta & \text { if } \quad q=-\frac{1}{2},-\frac{3}{2}, \cdots\end{cases}
$$

Now fix a point $x \in \boldsymbol{R}^{m} \backslash W$ and fix a chain $\gamma_{\sigma}(x)$ (resp. $\tilde{\gamma}_{\sigma}(x)$ ) as above. Since the simple real roots $\theta_{j}(x)(j=2 s+1, \cdots, m+1)$ vary continuously on $x$, we can 


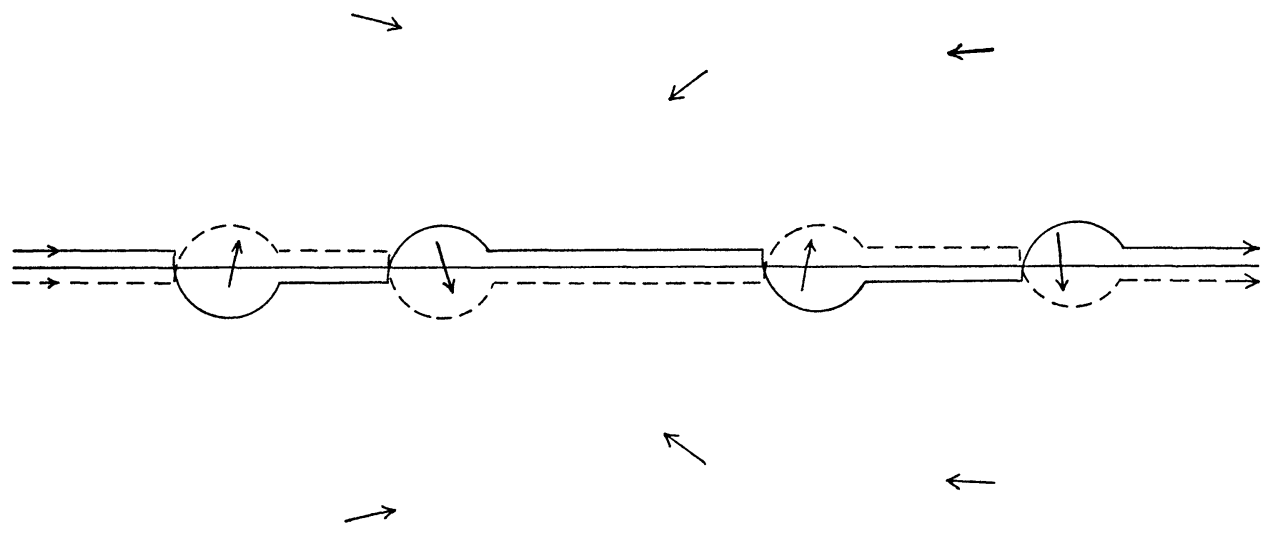

[Fig. 1] Arrows indicate the movements of roots when $\varepsilon$ vary from negative to positive values. The solid line is the chain for $\int_{V} \chi_{q}(\varphi(x, \theta)+i \varepsilon) d \theta$ and the dotted line is one for $\int_{V} \chi_{q}(\varphi(x, \theta)-i \varepsilon) d \theta$. The branches of these lines are taken so that $\varphi(x, \theta)^{q}$ take real positive values when $\theta \rightarrow+\infty$ on the chain.

take $\gamma_{\sigma}(y)$ (resp. $\left.\tilde{\gamma}_{\sigma}(y)\right)$ in common with $\gamma_{\sigma}(x)$ (resp. $\left.\tilde{\gamma}_{\sigma}(x)\right)$ provided that $y \in U$. Then we have

$$
\begin{aligned}
G_{q}^{\sigma}(y)= & \lim _{\varepsilon \downarrow 0} I_{\varepsilon}(y)=\int_{r_{\sigma}(x)} \chi_{q}(\varphi(y, \theta)) d \theta \\
& \left(\operatorname{resp} \cdot \int_{\tilde{r}_{\sigma}(x)} \chi_{q}^{\sigma}(\varphi(y, \theta)) d \theta\right) \quad \bmod C^{\omega}(U) .
\end{aligned}
$$

This proves the proposition.

\section{§3. Sharpness of $G_{q}^{\sigma}(x)$.}

In this section we shall represent a criterion for sharpness in terms of homology classes (Theorem 2). Theorem 1 and Corollary will then follow from this theorem. Certainly, if we restrict the problem on the sharpness to the $A_{m}$-type one, we can prove Theorem 1 without the homological formulation. But we shall need it to treat more general type of singularlities such that dim $\theta \geqq 2$ (c. f. [3], [5]).

Now take a point $x^{0} \in W$ and a component $\omega$ of $\boldsymbol{R}^{m} \backslash W$ with $x^{0} \in \partial \omega$. Our aim is to find a criterion for the sharpness of $G_{q}^{\sigma}(x)$ at $x^{0}$ from $\omega$. Take a point $x^{1} \in \omega$ and join $x^{0}$ to $x^{1}$ by a smooth path in $\omega$ :

$$
l: x=x^{t} \quad(0 \leqq t \leqq 1) .
$$

Here we assume $\left.x^{t}\right|_{t=1}=x^{1}$ and $\left.x^{t}\right|_{t=0}=x^{0}$. For each $x \in l$, put 


$$
\begin{aligned}
& \hat{Z}:=(l \times C) \backslash\{(x, \theta) ; \varphi(x, \theta)=0\}=\left\{(x, \theta) ; x \in l, \theta \in \hat{V}_{x}\right\}, \\
& \tilde{Z}:=\left\{(x, \theta) ; x \in l, \theta \in \tilde{V}_{x}\right\} .
\end{aligned}
$$

The chains $\gamma_{\sigma}(x)$ and $\tilde{\gamma}_{\sigma}(x)$ defined by Definition 2 determine homology classes

$$
\alpha_{\sigma}(x):=\left[\gamma_{\sigma}(x)\right] \in H_{1}\left(\hat{V}_{x}\right), \quad \tilde{\alpha}_{\sigma}(x):=\left[\tilde{\gamma}_{\sigma}(x)\right] \in H_{1}\left(\tilde{V}_{x}\right) .
$$

Further we define

$$
\alpha_{\sigma}^{*}(x):=i^{*} \alpha_{\sigma}(x) \in H_{1}(\hat{Z}), \quad \tilde{\alpha}_{\sigma}^{*}(x):=\tilde{i}^{*} \tilde{\alpha}_{\sigma}(x) \in H_{1}(\tilde{Z})
$$

as images of $\alpha_{\sigma}(x)$ and $\tilde{\alpha}_{\sigma}(x)$ under the maps

$$
i^{*}: H_{1}\left(\hat{V}_{x}\right) \longrightarrow H_{1}(\hat{Z}), \quad \tilde{i}^{*}: H_{1}\left(\tilde{V}_{x}\right) \longrightarrow H_{1}(\tilde{Z})
$$

where $i: \hat{V}_{x} \rightarrow \hat{Z}$ and $\tilde{i}: \tilde{V}_{x} \rightarrow \tilde{Z}$ are the inclusion maps. From know on we shall assume, for the path $l$, the following condition:

For any $x \in l, \alpha_{\sigma}(x)$ (resp. $\left.\tilde{\alpha}_{\sigma}(x)\right)$ has the same

image in $H_{1}(\hat{Z})\left(\operatorname{resp} . H_{1}(\tilde{Z})\right)$.

Though this assumption fails for general $l$, this is not so restrictive. In fact the condition is satisfied if we replace $l$ by its restriction to a sufficientry small neighbourhood of $x^{0}$. Now under the assumption $(*)$, we may write

$$
\alpha_{\sigma}^{*}:=\alpha_{\sigma}^{*}(x), \quad \tilde{\alpha}_{\sigma}^{*}:=\tilde{\alpha}_{\sigma}^{*}(x),
$$

and we have the following theorem.

THEOREM 2. Take and fix a pornt $x^{0} \in W$ and a component $\omega$ of $\boldsymbol{R}^{m} \backslash W$ with $x^{0} \in \partial \omega$. Further take $a$ path $l$ in $\omega$ with an endpornt $x^{0}$ which satisfies the assumption (*). Then if there is a class $\beta \in H_{1}\left(\hat{V}_{x 0}\right)$ (resp. $\left.\tilde{\beta} \in H_{1}\left(\tilde{V}_{x 0}\right)\right)$ such that

$$
\alpha_{\sigma}^{*}=\beta^{*} \text { in } H_{1}(\hat{Z})\left(\text { resp. } \tilde{\alpha}_{\sigma}^{*}=\tilde{\beta}^{*} \text { in } H_{1}(\tilde{Z})\right),
$$

then $G_{q}^{\sigma}(x)$ for $q=-1,-2, \cdots\left(\right.$ resp. $\left.q=-\frac{1}{2},-\frac{3}{2}, \cdots\right)$ are sharp at $x^{0}$ from $\omega$.

Proof. We shall consider the case when $q=-1,-2, \cdots$ (The other case is treated similary). Assume that there is a class $\beta \in H_{1}\left(\hat{V}_{x 0}\right)$ such that $\alpha_{\sigma}^{*}=\beta^{*}$. Then there is a sufficiently small $\varepsilon$ and a chain $\gamma$ in $\hat{V}_{x 0}$ such that

$$
\gamma=\gamma_{\sigma}\left(x^{t}\right) \quad \text { for all } t \text { with } 0 \leqq t \leqq \varepsilon \text { and }[\gamma]=\beta \text {. }
$$

Then we have that

$$
G(x) \equiv \int_{\gamma} \chi_{q}(\varphi(x, \theta) d \theta
$$

is analytic in a neighbourhood of $x=x^{0}$ and further 


$$
G(x)=\int_{\gamma_{\sigma}(x t)} \chi_{q}(\varphi(x, \theta)) d \theta=G_{q}^{\sigma}(x)
$$

in a neighbourhood of $\left\{x^{t} ; 0<t \leqq \varepsilon\right\}$. Thus, along the path $l$, we can continue $G_{q}^{\sigma}(x)$ analytically up to $x=x^{0}$ and this proves that $G_{q}^{\sigma}(x)$ is sharp at $x^{0}$ from $\omega$.

Proof of Theorem 1. Take $x^{0} \in W$ and $\omega$ as in Theorem 1. Further take a smooth path $l$ in $\omega$ with endpoints $x^{0}$ and $x^{1} \in \omega$, and consider the images of the roots $\theta_{j}(x), x \in l$;

$$
\begin{aligned}
& \Gamma_{1}:=\left\{\theta_{j}(x) ; x \in l, j=1,2, \cdots, 2 s\right\}, \\
& \Gamma_{2}:=\left\{\theta_{j}(x) ; x \in l, j=2 s+1, \cdots, m+1\right\} .
\end{aligned}
$$

Now assume that $q \in \boldsymbol{Z}, \sigma=+1$ and suppose that when $x \rightarrow x^{0}$ along the path $l$, only the combinations of [I] and [II] occur. Then $\Gamma_{1} \cap \boldsymbol{R}=\phi$ and $\Gamma_{2} \subset \boldsymbol{R}$. Here we note that we may replace $\gamma_{+}\left(x^{1}\right)$ by the difference of two chains $\gamma_{+}^{\prime}$ and $\gamma_{+}^{\prime \prime}$, where $\gamma_{+}^{\prime}$ is one enclosing all the imaginary $\operatorname{roots} \theta_{j}\left(x^{1}\right)$ with $\operatorname{Im} \theta_{j}\left(x^{1}\right)$ $>0$ in the positive direction, and $\gamma_{+}^{\prime \prime}$ is one enclosing all the imaginary roots $\theta_{j}\left(x^{1}\right)$ with $\operatorname{Im} \theta_{j}\left(x^{1}\right)<0$ in the positive direction. It is clear that $\left[\gamma_{+}\left(x^{1}\right)\right]=$ $\left[\gamma_{+}^{\prime}-\gamma_{+}^{\prime \prime}\right]$ in $H_{1}\left(\hat{V}_{x 1}\right)$. Further, by taking a restriction of $l$ if necessary, we may assume that $\gamma_{+}^{\prime}-\gamma_{+}^{\prime \prime}$ does not intersect $\Gamma_{1} \cup \Gamma_{2}$ and $\left[\gamma_{+}(x)\right]=\left[\gamma_{+}^{\prime}-\gamma_{+}^{\prime \prime}\right]$ in $H_{1}\left(\hat{V}_{x}\right)$ for all $x \in l$. Then the assumptions of Theorem 2 are satisfied and we have that $G_{q}^{+}(x)$ is sharp at $x^{0}$ from $\omega$. The other three cases will be treated by similar manners and this proves the theorem.

The corollary will follow directly from Theorem 1 .

Remark 1. The condition in Theorem 2 is a special case of the "local Petrowski condition" of L. Gårding [3].

Remark 2. The classes $\alpha_{\sigma}^{*}, \tilde{\alpha}_{\sigma}^{*}$ in Theorem 2 are determined if we take a path $l$. On the other hand, the sharpness is determined if we take $x^{0}$ and $\omega$. Thus it is desireble to formulate the criterion in terms of classes which are determined not by $l$ but by $x^{0}$ and $\omega$. Unfortunately the structure of $H_{1}(\hat{Z})$ and $H_{1}(\tilde{Z})$ are changed if we change $l$ and it seems to be difficult to set desired formulation.

Remark 3. In this paper, we have considered $G_{q}^{\sigma}(x)$ defined not by (1) but by (3). But in practice, it is the case when the region of integration $V$ is not $\boldsymbol{R}$ (or $\boldsymbol{R}^{N}$ ) but some open neighbourhood of the origin in $\boldsymbol{R}$ (or $\boldsymbol{R}^{N}$ ). In these cases, it may necessary to consider, instead of $H_{1}\left(\hat{V}_{x}\right)$ etc., some relative homology groups. But since we throughly consider our problems modulo $C^{\omega}-$ (or $C^{\infty}-$ ) functions, no essential differences may appear.

Remark 4. Concerning to $A_{m}$-singularities, in some sense, our Corollary covers fairly general situations. In fact, for any $x^{0} \in W$, we can reduce the 
problem of sharpness at $x^{0}$ for $A_{m}$-type integral to one at the origin for $A_{k}$ type integral $(0 \leqq k \leqq m)$ (see $[1],[5]$ ). From this point of view, we may say that the only interest is in the problem of sharpness at the origin.

Remark 5. In Corollary, (a) is the case when $\alpha_{\sigma}^{*} \neq 0$ (though it vanishes as a relative cycle) and (b) is the case when $\alpha_{\sigma}^{*}=0$. Thus in the case (b) $G_{q}^{\sigma}(x)$ has a strong lacuna in $\omega$, that is, $G_{q}^{\sigma}(x) \equiv 0$ in $\omega$. Since $\varphi(x, \theta)$ is a real polynomial, the case (b) may occur only if $m+1$ is even, i. e. $\operatorname{dim} x=m$ is odd.

Finally we state two simple examples.

Example 1. $(m=2)$ By $\varphi(x, \theta)=\frac{\partial \varphi}{\partial \theta}(x, \theta)=0$, we have $4 x_{1}^{3}+27 x_{2}^{2}=0$. Then we have [Fig. 2].

Example 2. $(m=3)$ The zero locus of $\varphi(x, \theta)=\frac{\partial \varphi}{\partial \theta}(x, \theta)=0$ is the "swallow's tail". [Fig. 3] represents the sections of the zero locus at $x_{1}=c_{1}>0$ and $x_{1}=c_{2}<0$.

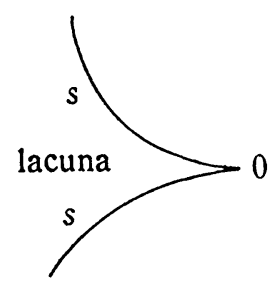

$q \in Z, \sigma=1$.

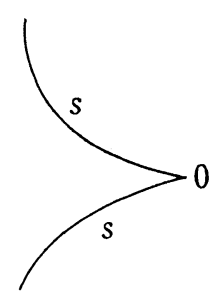

$q \in Z, \sigma=-1$.

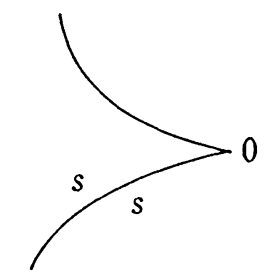

$q \in Z+1 / 2, \quad \sigma=1$.

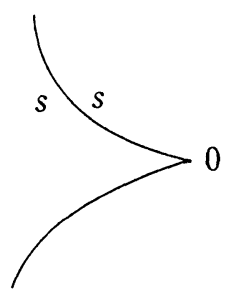

$q \in \boldsymbol{Z}+1 / 2, \quad \sigma=-1$.

[Fig. 2] Sharp fronts at a cusp. 


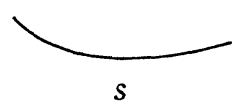

lacuna
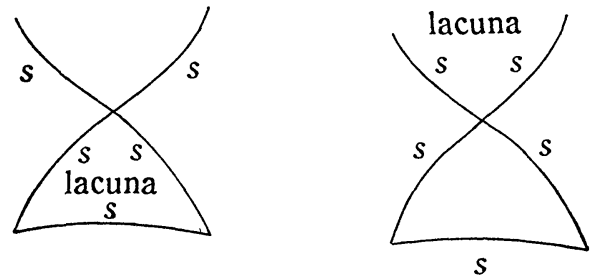

$q \in Z, \sigma=1$.

$q \in Z, \sigma=-1$.
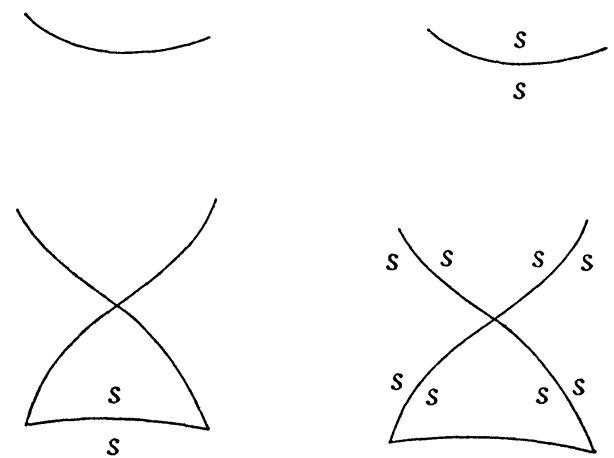

$q \in \boldsymbol{Z}+1 / 2, \sigma=1$.

$q \in Z+1 / 2, \sigma=-1$.

[Fig. 3] Sharp fronts at a swallow's tail.

\section{REFERENCES}

[1] Duistermaat, J.J., Oscillatory integrals, Lagrange immertions and unfolding of singularities, Comm. Pure Appl. Math. 27 (1974), 207-281.

[2] FEDORYUK, M.V., Singularities of the kernels of Fourier integral operators and the asymptotic behaviour of the solution of the mixed problem, Russ. Math. Surv. $32: 6,(1977), 67-120$.

[3] GÅRDING, L., Sharp fronts of paired oscillatory integrals, Publ. RIMS Kyoto Univ. 12 Suppl. (1977) 53-68.

[4] Yamada, H., Sharpness of parametrices for strictly hyperbolic operators, Proc. Japan Acad. 58 (1982), 137-140.

[5] Yamada, H., Sharp fronts of parametrices for some hyperbolic operators, in preparation.

Department of Mathematics

Miyagi University of Education

Aramaki Aoba, Sendai, 980, Japan. 